\title{
Explicaciones, concepciones y obstáculos sobre el origen de las especies en estudiantes de Escuela Secundaria de Argentina
}

\section{Explanations, conceptions and obstacles about the origin of species in High School students in Argentina}

\author{
Leonardo Martín González Galli ${ }^{1}$ \\ https:// orcid.org/0000-0002-0713-164X \\ Gastón Mariano Pérez ${ }^{2}$ \\ https://orcid.org/0000-0001-9751-2042 \\ Cinthia Karen Alegre ${ }^{3}$ \\ https:// orcid.org/0000-0002-6037-6857 \\ Sebastian Joelson Escoto ${ }^{2}$ \\ https://orcid.org/0000-0001-8013-8410
}

\begin{abstract}
Resumen: Aunque existen numerosas investigaciones que analizan el modo en que los estudiantes explican la evolución adaptativa, son escasos los estudios en que se aborda cómo explican el origen de nuevas especies. En este trabajo caracterizamos, en base a una metodología cualitativa interpretativa, el modo en que estudiantes de una escuela de nivel secundario de Argentina explican el origen de nuevas especies. A tal fin utilizamos un cuestionario abierto en el que pedimos a los estudiantes explicar el origen y extinción de ciertas especies. A partir de las respuestas identificamos dos patrones explicativos basados en la noción de adaptación y un tercero en la de hibridación. Interpretamos estas respuestas en términos de una jerarquía representacional que incluye desde las ideas más específicamente ligadas a los casos discutidos hasta los obstáculos epistemológicos que constituyen los modos de pensamiento más generales subyacentes.
\end{abstract}

Palabras clave: Escuela secundaria. Enseñanza de biología. Evolución biológica.

\begin{abstract}
Although there exists a great deal of research that analyzes the way in which the students explain adaptive evolution, few studies address how they explain the origin of new species. In this work, we characterize, based on a qualitative interpretative methodology, the way in which students in a secondary school in Argentina explain the origin of new species. For this purpose we use an open questionnaire in which we ask the students to explain the origin and extinction of certain species. From the answers we identified two explanatory patterns based on the notion of adaptation and a third on the hybridization. We interpret these responses in terms of a representational hierarchy ranging from the ideas most specifically linked to the cases discussed to the epistemological obstacles that constitute the most general modes of thought underlying.
\end{abstract}

Keywords: High school. Biological teaching. Biological evolution.

\footnotetext{
${ }^{1}$ Consejo Nacional de Investigaciones Científicas y Técnicas (CONICET), UBA, Buenos Aires, Argentina. E-mail: <gonzalezgalli@cefiec.fcen.uba.ar>.

${ }^{2}$ Universidad de Buenos Aires (UBA), Facultad de Ciencias Exactas y Naturales (CEFIEC), Buenos Aires, Argentina.

${ }^{3}$ Instituto Superior de Formación Docente $N^{\circ} 41$, Adrogué, Argentina.
} 


\section{Introducción}

Existe un amplio consenso sobre la gran importancia de la teoría de la evolución (TE) en la enseñanza de las ciencias para el nivel general y obligatorio que se basa en el lugar central de dicha teoría en la biología y en su gran influencia en la cultura general (STAMOS, 2008). También existe un fuerte consenso sobre el hecho de que el aprendizaje de estos contenidos resulta extremadamente dificultoso por diversos motivos, entre los cuales se ha destacado la existencia de concepciones erróneas fuertemente resistentes al cambio mediante la instrucción (KAMPOURAKIS, 2014). La mayoría de las investigaciones sobre este tema se caracterizan por el uso de instrumentos que buscan detectar concepciones previamente definidas por el investigador (véase, por ejemplo, ANDERSON; FISHER; NORMAN, 2002). Otra característica de la investigación en esta área es el diseño y prueba de unidades didácticas que tienen por objetivo principal transformar dichas concepciones mediante intervenciones basadas en alguna versión de la teoría del cambio conceptual (véase, por ejemplo, JIMÉNEZ ALEIXANDRE, 1991). Por otro lado, la mayoría de estas investigaciones se centra en las explicaciones de los estudiantes en relación con la adaptación biológica y sólo en pocas ocasiones se ocupan de las explicaciones sobre el origen de las especies (véase, por ejemplo, ANDERSON; FISHER; NORMAN, 2002). Solo unas pocas investigaciones han explorado este tópico de un modo específico (ver EVANS, 2000; SAMARAPUNGAVAN; WIERS, 1997).

La investigación que reportamos aquí tiene varios rasgos en alguna medida originales en relación con la mayoría de las investigaciones precedentes sobre el tema. En primer lugar, nos centramos en las explicaciones sobre el origen de las especies o especiación (y, secundariamente, en el concepto de especie) y no de las adaptaciones (tema que abordamos en un trabajo anterior) (GONZÁLEZ GALLI; MEINARDI, 2015). En segundo lugar, nuestra aproximación es más "inductiva" en el sentido de que no buscamos evaluar si los estudiantes tienen cierta concepción errónea previamente definida sino que enfrentamos a los estudiantes a preguntas abiertas que exponen el fenómeno a explicar. Por último, nuestro enfoque no apunta a desarrollar estrategias didácticas tendientes al reemplazo de las concepciones de los estudiantes sino que enfatizamos el desarrollo de las destrezas metacognitivas como condición para una autorregulación de sus modos de pensar. Por tal motivo, ofrecemos un análisis en términos de obstáculos epistemológicos que sirva de insumo para favorecer la toma de conciencia y la regulación de estas formas de pensar que, en ciertos momentos, pueden dificultar la construcción de los modelos científicos escolares.

El objetivo específico de este trabajo es caracterizar las explicaciones de estudiantes de una escuela secundaria de Argentina sobre el origen de las especies en términos de una jerarquía representacional que incluye desde las estructuras mentales más específicas y explícitas que llamamos "patrones explicativos" a aquellas más generales e implícitas que denominamos "obstáculos", llamando simplemente "concepciones" a aquellas ideas que se encuentran entre ambos extremos. 
Explicaciones, concepciones y obstáculos sobre el origen ...

\section{Marcos teóricos}

\section{Teoría de la evolución}

La evolución abarca un amplio conjunto de fenómenos y la TE incluye diversos modelos, cada uno de los cuales pretende dar cuenta de alguno de estos fenómenos (LLOYD, 1994; THOMPSON, 1989). Desde el punto de vista didáctico importa acotar e identificar con claridad qué fenómenos esperamos que los estudiantes puedan abordar con los modelos enseñados. Asumimos aquí que las dos grandes cuestiones que busca explicar la TE son: (1) el origen de la adaptación biológica y (2) el origen de la diversidad biológica (STERELNY; GRIFFITHS, 1999). En cuanto a qué modelos resultan centrales para dar cuenta de estos fenómenos sugerimos que el modelo de evolución por selección natural (MESN) ocupa un lugar de privilegio (GOULD, 2004). Siendo conscientes de que existen muchos más modelos dentro de la actual TE consideramos que el MESN constituye la base que todo estudiante debería aprender para construir una primera explicación para el origen de la adaptación y de nuevas especies (GONZÁLEZ GALLI; MEINARDI, 2013).

\section{El concepto de obstáculo}

El concepto de obstáculo en la didáctica de las ciencias naturales está "inspirado" en el de obstáculo epistemológico de Bachelard (2004), que se refería a formas de pensar muy arraigadas en la comunidad científica y que dificultaban el avance del conocimiento al impedir la adopción de nuevos puntos de vista potencialmente más fructíferos. La visión de Bachelard sobre estos modos de pensar es totalmente negativa: se avanza contra ellos. En la didáctica la perspectiva es más positiva porque se reconoce que estos estilos de razonamiento al mismo tiempo posibilitan y limitan el aprendizaje (ASTOLFI, 1999). Adoptamos una definición precisa de obstáculo que hemos propuesto en otro trabajo (GONZÁLEZ GALLI; MEINARDI, 2016) según la cual un obstáculo es cualquier concepción que cumpla con tres características: (1) transversalidad (subyace a diversas concepciones más específicas), (2) funcionalidad (le permite al sujeto explicar ciertos fenómenos) y (3) conflictividad (puede dificultar el aprendizaje del modelo científico que se pretende enseñar). Según esta definición los obstáculos son una parte constitutiva y funcional de la estructura cognitiva del sujeto y, por lo tanto, se los considera no eliminables. Otros autores han arribado a nociones análogas en sus intentos de caracterizar los razonamientos subyacentes a las concepciones de los estudiantes. Tal es el caso del concepto de restricción cognitiva (TALANQUER, 2009) y de constructo cognitivo (cognitive construal) (COLEY; TANNER, 2012). El objetivo didáctico no sería el remplazo de los obstáculos por otras formas de pensar sino que los estudiantes sean conscientes de que tienden a pensar de ese modo y de que sean capaces de regular dichos pensamientos. Estos objetivos didácticos se centran en el desarrollo de las capacidades metacognitivas de los estudiantes, motivo por el cual decimos que el objetivo último es que estos desarrollen una "vigilancia metacognitiva" sobre sus obstáculos.

\section{Enfoque metodológico}

La población con la que trabajamos consta de sesenta estudiantes de segundo año (con una edad promedio de quince años) de una escuela secundaria de gestión estatal de la Ciudad 
Autónoma de Buenos Aires (Argentina). Los estudiantes participantes no habían recibido instrucción formal en genética ni evolución. Para la toma de datos utilizamos un cuestionario (ver anexo) que consta de cuatro preguntas abiertas. El diseño del instrumento fue validado por la revisión de tres investigadores. Todos los estudiantes respondieron individualmente y por escrito el cuestionario. Además, realizamos diecisiete entrevistas semiestructuradas individuales en las que el diálogo se basó en la aclaración y ampliación de las respuestas escritas al cuestionario. Nuestro enfoque metodológico es cualitativo interpretativo (LATORRE BELTRÁN; RINCÓ IGEA; ARNAL, 1997). Nos basamos en la teoría fundamentada y, más específicamente, en el método comparativo constante (GLASER; STRAUSS, 1967) para generar categorías teóricas que pretenden dar cuenta de las concepciones de los estudiantes. Para incrementar la validez de los análisis se llevó a cabo una triangulación de investigadores.

\section{Resultados y análisis}

A continuación se presenta el análisis de las respuestas a cada problema. En dicho análisis generamos un conjunto de categorías teóricas que pretenden representar las concepciones utilizadas por los estudiantes en sus respuestas. Luego, en la sección “discusión” caracterizaremos los patrones explicativos, en los que se articulan varias concepciones, para finalmente proponer los posibles obstáculos epistemológicos subyacentes.

\section{Problema 1}

El objetivo de esta primera pregunta era indagar a qué tipo de proceso atribuyen el origen de las especies los estudiantes. Llamaremos "por generación espontánea" a la opción A, "creacionista" a la B y "evolucionista" a la C. La mayoría de los estudiantes eligió la opción evolucionista $(\mathrm{C})$, un porcentaje menor supuso un origen por generación espontánea (A) y muy pocos eligieron la opción creacionista (B). Un número considerable eligió más de una opción (Tabla 1).

Tabla 1. Porcentaje de estudiantes que eligieron cada opción en la pregunta 1

\begin{tabular}{clc}
\hline \multicolumn{1}{c}{ Opción } & $\begin{array}{c}\text { Porcentaje de estudiantes } \\
\text { que eligieron cada opción }\end{array}$ \\
\hline A & Cada especie surgió de un modo natural e independiente. & 8.33 \\
B & Cada una de estas especies fue creada por algo o alguien. & 5 \\
C & Cada especie surgió por modificación de otra especie anterior. & 60 \\
& Más de una opción & 26.66 \\
\hline
\end{tabular}

Fuente: elaborada por los autores.

Un resultado importante es que muy pocos estudiantes elijen la opción creacionista (B) y aquellos que lo hicieron justificaron su elección afirmando que las nuevas especies no pueden venir de "de la nada" pero sin explicitar una justificación basada en la creación divina. Para esta opción y la (A) no encontramos ninguna concepción recurrente en la justificación. 
En las argumentaciones de la opción (C) encontramos varias concepciones. A partir del análisis interpretativo construimos seis categorías. A continuación definimos dichas categorías, señalamos (entre paréntesis) el porcentaje de respuestas en que aparecen y ofrecemos un ejemplo de respuesta en que se expresan:

1 Evolución (16,66\%). En estos casos el sujeto supone que la evolución implica per se el origen de nuevas especies. Ejemplo: "Se sabe que estas especies fueron evolucionando hasta llegar a convertirse en lo que son ahora".

2 Cambio individual adaptativo (23,33\%). Se supone que la adaptación al ambiente implica el origen de una nueva especie. Ejemplo: "Porque evolucionan y se adaptan al lugar.". El hecho de que el cambio se dé a nivel individual es a veces explícito, por ejemplo: "Porque creo que los individuos fueron cambiando con los años.". Muchas veces, sin embargo, aunque esto no es explícito, inferimos que el cambio adaptativo se entiende como una transformación individual. Esto lo sugiere el uso de los términos acostumbramiento y mutación, por ejemplo: "Porque nuestros genes van mutando para adaptarse al nuevo hábitat y forma de vida." y "Cada especie surgió por modificación porque cada especie para sobrevivir tiene que ratificar [sic] sus necesidades. Tenian que acostumbrarse a su bábitaty asi se fueron transformando". 3 Reproducción (10\%). Las nuevas especies derivarían de eventos de reproducción (frecuentemente referidos con expresiones como "se cruzaron con" o "se mezclaron con"). En estas respuestas no se diferencian los niveles individual y poblacional de análisis. Así, no es claro qué significa decir que una especie "se reproduce". En general (las respuestas a las entrevistas apoyan esta interpretación) se supone que dicha "reproducción" se da entre individuos de diferentes especies (hibridación). Interpretamos que subyace aquí una analogía entre el individuo y sus propiedades (tal como la de reproducirse) y una entidad supraindividual como es la especie. Ejemplo: "Porque las especies se reproducen y puede nacer alguna especie con alguna 'deformación' que sería para sobrevivir." y "Porque cada especie se puede alterar o surgir una nueva si hay una cruza entre dos especies diferentes.".

4 Finalismo (18,33\%). Las nuevas especies surgen para cumplir algún fin predeterminado que es expresado de manera más o menos explícita en la respuesta. Ejemplo: "Porque al principio solo existía una especie de ser vivo, luego fue evolucionando para convertirse en especies diferentes.".

5 Necesidad (8,33\%). Las especies se modifican adaptativamente según sus necesidades de supervivencia y dicho cambio implica el origen de una nueva especie. Ejemplo: "Por la evolución de los seres según las necesidades y el ambiente en el que se encuentran.".

6 Analogía con la especie bumana (6,66\%). Se asume que como nuestra especie evolucionó, lo mismo debe ser válido para las demás especies y que dicho cambio implica el origen de nuevas especies. Ejemplo: "Porque como el humano fue evolucionando por medio de pequeñas modificaciones tras millones de años, supongo que otros seres vivos también evolucionaron de esta manera.".

En las respuestas a las preguntas siguientes encontramos varias de las mismas concepciones identificadas para la respuesta 1, por lo que solo comentaremos aquellas concepciones diferentes y específicas identificadas en las respuestas a estas preguntas. 


\section{Problema 2}

Este problema tenía como objetivo indagar qué aspectos de un organismo deberían cambiar para ser considerado de otra especie, más específicamente, se trata de indagar en qué medida el concepto de especie implícito en las explicaciones de los estudiantes está basado en la homogeneidad morfológica. La Tabla 2 muestra los porcentajes de elección para esta pregunta.

Tabla 2. Porcentaje de estudiantes que eligieron cada opción en la pregunta 2

\begin{tabular}{ccc}
\hline \multicolumn{1}{c}{ Opciones } & $\begin{array}{c}\text { Porcentaje de estudiantes } \\
\text { que eligieron cada opción }\end{array}$ \\
\hline A & Creo que no cambiarían, serían iguales. Seguirían siendo ballenas. & 5 \\
B & Creo que cambiarían pero seguirían siendo ballenas. & 53.33 \\
C Creo que cambiarían y ya no serían ballenas, serían otra especie. & 23.33 \\
\multicolumn{2}{c}{ Elige más de una opción } & 13.33 \\
No acuerda con ninguna opción & 5 \\
\hline
\end{tabular}

Fuente: elaborada por los autores.

La opción fijista (A) es elegida por muy pocos estudiantes (3). Así, la gran mayoría $(90 \%)$ asume una perspectiva evolucionista (opciones B y C). Aproximadamente la mitad de los sujetos $(53,33 \%)$ supone que cambiarán pero que seguirán siendo ballenas (opción B). En las justificaciones a esta elección aparecen varias de las concepciones identificadas en el problema 1: adaptación (en 10 respuestas), finalismo (en 6), necesidad (en 3) y reproducción (en 1). Interpretamos que esta elección expresa una concepción según la cual la categoría de especie es fija, es decir, hay cambio fenotípico pero dicho cambio no supone un cambio de especie. Para dar cuenta de esta concepción definimos la categoría 7 que denominamos estabilidad específica. Por ejemplo, "Porque a menos de que se extinga la especie, cambiarían, habrán cruces con otras especies, pero las ballenas seguirian siendo ballenas" y "Cambiaron por abi su forma, pero seguirían siendo ballenas". A partir de las respuestas dadas por el 23,33\% que eligió la opción C definimos la categoría 8 que denominamos cambio profundo (15\% del total de respuestas) según la cual cuando el cambio supera cierto umbral de magnitud entonces sí implica un cambio de especie, por ejemplo " $A l$ gunas ballenas podrían cambiar tanto que llegarían a formar otro animal o especie". Esta concepción se expresa incluso en justificaciones a la opción B, como vemos en el siguiente ejemplo: "Porque si una especie cambia demasiado pasa a denominarse de otra manera, con otro nombre. Cambiarían porque la especie evolucionaría". En síntesis, de los resultados obtenidos para el problema 2 se infiere que muchos estudiantes asumen que las especies cambiarán pero que seguirán perteneciendo a la misma especie. Por otro lado, consideran que solo cabe hablar de un cambio de especie cuando los rasgos que cambian son especialmente relevantes o cuando el cambio es de gran magnitud. Además, la magnitud del cambio sería proporcional al tiempo transcurrido. Las respuestas a las entrevistas apoyan estas interpretaciones, por ejemplo:

I [investigador]: "La segunda pregunta sería si a ese animal que sufrió todos esos cambios vos le seguirías llamando 'ballena' o si lo considerarias...". 
E [estudiante]: "No, le cambiaría el nombre, si es tipo 10 millones de años ya no se llamaria 'ballena', se llamaria de una forma diferente, tendría procedencia de las ballenas, pero no sería con ese nombre".

No encontramos aquí indicios claros de que estén pensando en términos de divergencia sino más bien de cambio anagenético ${ }^{4}$, en el que la nueva especie reemplaza a la especie ancestral de la que deriva. Algunos estudiantes son explícitos al respecto, por ejemplo "Yo [...] creo que cuando un animal se aparea con otro animal crea una especie nueva. Y cuando los creadores de la nueva especie mueren, mueren con ellas esas especies y queda la nueva". En el mismo sentido, una estudiante afirma que "Habría una especie evolucionada que tiene procedencia de las ballenas [...], habrian desaparecido o estarian prácticamente extintas porque, al haber una nueva especie más evolucionada y apta, la especie que hoy conocemos sería 'obsoleta". Es decir, habría un reemplazo de la especie anterior por una nueva y más "apta".

\section{Problema 3}

El objetivo de esta pregunta era indagar cómo explican los estudiantes la variación en el número de especies a lo largo del tiempo. Se trata de un modo indirecto de preguntar cómo y por qué surgen nuevas especies y se extinguen las ya existentes. En relación con el aumento del número de especies encontramos las mismas concepciones identificadas en el caso de la pregunta 1 , por ejemplo " $A$ lo largo del tiempo fueron evolucionando para tratar de sobrevivir en nuevos hábitats" y "Por abi esos felinos se adaptaron a otros climas y eso les produjo cambios en el fisico". En ambos casos interpretamos que hay expresiones de las categorías cambio individual adaptativo y finalismo. También encontramos numerosas (35\%) expresiones de la concepción 3 (reproducción), por ejemplo "Quizá porque se mezclaron distintas especies de animales y cuando bubo varios felinos diferentes estos se mezclaron entre ellos y fueron cada vez más [especies felinas]" y "Seguramente se fueron reproduciendo, pero no solo con el mismo animal, puede ser con otro, porque si no saldría el mismo". Las categorías específicas aparecieron con la siguiente frecuencia: reproducción (35\%); cambio individual adaptativo (26,66\%); finalismo (20\%); evolución (13,33\%) y necesidad (3,33\%). Algunas respuestas evidencian una idea según la cual las nuevas especies surgirían como consecuencia de la adaptación local de distintas poblaciones a diferentes ambientes. Denominamos divergencia geográfica (categoría 9) (15\%) a esta concepción que resulta especialmente interesante por estar relacionada con el modelo de especiación alopátrico, según el cual las nuevas especies surgen como consecuencia de la evolución de alguna barrera reproductiva entre dos poblaciones gracias a la interrupción del flujo génico debida a una barrera física (FUTUYMA, 2009). Este es el modelo de especiación más aceptado y cuyo aprendizaje sería uno de los principales objetivos de la enseñanza de la biología

\footnotetext{
${ }^{4}$ Se denomina anagénesis al proceso evolutivo que supone el cambio de un linaje sin que tenga lugar un evento de divergencia evolutiva. Cuando sucede esto último de habla de cladogenésis (FUTUYMA, 2009). Adoptando un concepto tipológico de especie (basado en la semejanza morfológica) los procesos anagenéticos pueden producir una nueva especie, que no sería más que una versión posterior y fenotípicamente muy modificada de la original. En cambio, si adoptamos el concepto biológico de especie (basado en el aislamiento reproductivo) solo un proceso cladogenético podría - por definición - generar una nueva especie.
} 
evolutiva. Un ejemplo de respuesta en que aparece esta concepción es: "Porque hay más mutaciones de este felino para los diferentes ecosistemas. Digamos que uno de estos felinos originales se va a la nieve; entonces con el paso del tiempo su descendencia tendría otras características como más pelo, otro color de pelaje para camuflarse... Claro, estamos hablando de un proceso de millones de años". En relación con las explicaciones sobre la extinción de especies definimos la categoría 10 (insuficiencia adaptativa) (26,66\%) según la cual la causa de la extinción reside en una suerte de "falla de la adaptación", es decir, por la gran magnitud o velocidad de un cambio ambiental algunas especies no logran adaptarse adecuadamente, por ejemplo "Porque algunas no lograron adaptarse a los nuevos territorios" y "Porque no se habrán podido adaptar a las condiciones de vida que cambiaron en el planeta [depredadores, clima, etc...]". En muchas otras respuestas, esta idea está implícita, por ejemplo "Los animales se adaptan y muchas de las especies que existieron no sobrevivieron a la 'era de bielo' u otros cambios climáticos, de depredación, o por falta de comida, etc.". Consideramos que estas explicaciones son coherentes con las respuestas a la pregunta 1) (y a las explicaciones del incremento en el número de especies en la pregunta 3) debido a que se basan en la noción de adaptación. También en relación con el problema de la extinción definimos la categoría 11 (causa antropogénica) que se expresa en muchas explicaciones $(30 \%)$ basadas en la intervención humana como causa de la extinción de las especies, por ejemplo "Porque actualmente el ser humano irrumpió en muchos ambientes naturales provocando asi la extinción de especies." y "Porque la mano del hombre destruye todo". En síntesis, encontramos que muchas respuestas en relación con el origen de nuevas especies se basan en las nociones de cambio individual adaptativo o de reproducción (en general, hibridación) mientras que las respuestas en relación con la reducción del número de especies se fundan en la idea de insuficiencia adaptativa o bien de causa antropogénica.

\section{Problema 4}

El objetivo de esta pregunta era conocer la importancia relativa del parecido morfológico para la concepción de especie de los estudiantes. Los resultados se muestran en la Tabla 3.

Tabla 3. Porcentaje de estudiantes que consideran que las figuras comparadas pertenecen a la misma especie

\begin{tabular}{lcccc}
\hline \multirow{2}{*}{ Ítem de la pregunta 4 } & \multicolumn{4}{c}{ Porcentaje de respuestas para cada opción } \\
\cline { 2 - 5 } & $\begin{array}{c}\text { Pertenecen a la } \\
\text { misma especies }\end{array}$ & $\begin{array}{c}\text { Pertenecen a } \\
\text { especies diferentes }\end{array}$ & $\begin{array}{c}\text { Ambas } \\
\text { opciones }\end{array}$ & $\begin{array}{c}\text { No sabe / } \\
\text { no responde }\end{array}$ \\
\hline Punto 1 (A/B) & 61,66 & 35 & 1,66 & 1,66 \\
Punto 2 (B/C) & 95 & 1,66 & 3,33 & 0 \\
Punto 3 (C/D) & 45 & 51,66 & 1,66 & 1,66 \\
Punto 4 (A/D) & 43,33 & 51,66 & 5 & 0 \\
\hline
\end{tabular}

Referencias: A/B: comparación entre dibujo A y dibujo B; B/C: comparación entre dibujo B y dibujo C; C/D: comparación entre dibujo $\mathrm{C}$ y dibujo D; A/D: comparación entre dibujo A y dibujo D.

Fuente: elaborada por los autores. 
Inferimos a partir de estos resultados que a mayor semejanza morfológica mayor probabilidad de que los estudiantes asignen los esquemas a una misma especie: la asignación a una misma especie es máxima (95\%) cuando los dibujos son idénticos (punto 2) y mínima cuando la diferencia entre los dibujos es máxima (puntos 3 y 4), con un valor intermedio cuando la diferencia también es intermedia (punto 1).

\section{Discusión}

\section{Patrones explicativos}

Una primera conclusión es que casi ningún estudiante supone un origen creacionista para las nuevas especies, resultado que contrasta con el énfasis puesto en el factor religioso en investigaciones realizadas en EUA (ALMEIDA, 2012; COSTA; MELO; TEIXEIRA, 2011; SMI$\mathrm{TH}, 2010)$. Dicha diferencia puede interpretarse como una consecuencia de la gran influencia en el público general de ese país del movimiento autodenominado "creacionismo científico" (o su más reciente versión la "teoría del diseño inteligente") (KAMPOURAKIS, 2014), no existiendo (por el momento) algo comparable en magnitud en Argentina. Es esperable, sin embargo, que la relevancia de este factor dependa fuertemente de la población concreta de que se trate. Selles, Dorville y Pontual (2016) señalan el crecimiento de este problema en Brasil.

Con respecto al problema del origen, los estudiantes producen explicaciones finalistas en las que el cambio que origina la nueva especie es individual y adaptativo. Para que dicho cambio dé origen a una nueva especie debe superar cierto umbral de magnitud (ver categoría 8). Este sería uno de los patrones explicativos más comunes y lo denominamos especiación por cambio adaptativo anagenético profundo (EAAP). Este patrón se expresa en respuestas como "Porque se van acostumbrando y adaptando al lugar y asi surgen los animales y las especies [...]". Las respuestas basadas en la categoría 9 - entre otras - podrían considerarse como la expresión de un segundo patrón explicativo que llamamos especiación adaptativa cladogenética (EAC). Este patrón comparte muchos supuestos con el EAAP (siempre se trata de un cambio adaptativo) pero tiene una importante diferencia dada por el hecho de que la idea de segregación espacial implica la noción de divergencia (cladogénesis), de gran relevancia conceptual (por ser parte del modelo de especiación alopátrico) y ausente en la explicaciones tipo EAAP. Un ejemplo de EAC sería "Distintos individuos de esa primera especie se pudieron haber trasladado a otras zonas [más frías o más cálidas] por lo que tuvieron que adaptarse al ambiente al que habian llegado y, por lo tanto, evolucionaron de manera distinta y formaron una especie nueva”. Identificamos un tercer patrón explicativo (ligado a la categoría 3) que llamamos especiación por hibridación (EH) según el cual las nuevas especies surgen de eventos de reproducción ("cruce" o "mezcla") entre especies preexistentes, por ejemplo "Puede ser que dos especies diferentes se mezclen y formen otra especie nueva".

\section{Relación entre patrones explicativos, concepciones y obstáculos}

Sugerimos que los patrones explicativos identificados se construyen relacionando diversas concepciones que, a su vez, pueden interpretarse como la expresión de varios obstáculos epistemológicos que hemos caracterizado en estudios previos (GONZÁLEZ GALLI; MEINARDI, 
2016). Las principales concepciones que se articulan en las explicaciones de tipo EAAP y EAC son la 2, 4 y 5 . En estas explicaciones se asume un cambio individual y adaptativo (concepción 2), en respuesta a una necesidad (concepción 5) y la direccionalidad (la orientación a la adaptación al servicio de la supervivencia) implica una finalidad (concepción 4). La diferencia entre la idea de EAAP y EAC reside en que en el segundo caso los estudiantes recurren también a la concepción 9 (divergencia geográfica). Las explicaciones de tipo EAAP pueden interpretarse como una extrapolación de las explicaciones que los estudiantes suelen utilizar para dar cuenta de la evolución adaptativa anagenética. Por el contrario, las explicaciones de tipo EH y EAC parecen ser un intento original de ofrecer una explicación ad hoc que dé cuenta específicamente del fenómeno de especiación. En relación con las concepciones que aparecen articuladas en las explicaciones de tipo EH la principal es la 3 (reproducción), ya que en general se habla de individuos de especies diferentes que se reproducen y se asume que dicha reproducción da origen a una nueva especie. En muchos casos, el "cruce" entre especies es la respuesta a una necesidad adaptativa, por lo que también están implicadas las concepciones 2, 4 y 5 .

En cuanto a los posibles obstáculos subyacentes, tal vez el más evidente sea el que llamamos teleología de sentido común (TSC) (GONZÁLEZ GALLI; MEINARDI, 2011; INAGAKI; HATANO, 2006). En efecto, las concepciones 2, 4 y 5, que se articulan en las explicaciones de tipo EAAP y EAC, implican razonamientos teleológicos. En el caso del cambio individual adaptativo (categoría 2) el cambio se da siempre en el sentido que maximiza el desempeño del organismo en el medio, lo que constituye el fin implícito del cambio. Más específicamente, la noción de acostumbramiento (incluida en la categoría 2) se basa en una analogía con el proceso de acomodación fisiológica, conductual o psicológica del individuo humano, proceso que siempre tiende a incrementar el ajuste al medio. La categoría 4 incluye las expresiones más explícitas en las que se menciona el fin que guía el proceso de cambio y la noción de necesidad (categoría 5) es intrínsecamente teleológica ya que solo una entidad que persigue algún fin puede tener una necesidad; lo que se necesita se necesita siempre para algo.

Otro posible obstáculo subyacente es el razonamiento centrado en el individuo (RCI) (GONZÁLEZ GALLI; MEINARDI, 2015), según el cual todo cambio se reduce a cambios a nivel individual. Las expresiones más claras de este obstáculo las constituyen las concepciones definidas por las categorías 2 y 3 . En ambos casos, el razonamiento se basa en una analogía entre un individuo y una entidad supraindividual (la especie). En el caso de la categoría 3, por ejemplo, algunos estudiantes hablan de que una especie "se reproduce (o se cruza o mezcla)", siendo que, en rigor, son los individuos los que se reproducen. No queda claro, en este caso, qué significaría exactamente que una especie "se reproduzca", pero la analogía permite de hecho dar cuenta del incremento en el número de especies: así como la reproducción de los individuos genera nuevos individuos, la reproducción de las especies generaría nuevas especies. La categoría 2 supone, por definición, un análisis a nivel individual.

En relación con las explicaciones de tipo EH, sugerimos que podría tratarse de otra expresión del razonamiento centrado en el individuo, ya que frecuentemente se expresa en términos de "reproducción entre especies", lo que supone la ya mencionada analogía entre el individuo y la especie. Por ejemplo (entrevista):

I: Bien, y esto que "fueron uniones de distintos animales" ¿A qué se refiere? ¿Podés dar un ejemplo? 
E: Por ejemplo un perro salchicha con un perro de raza, que al juntarse sale una mezcla de los dos.

I: Ajá, y ese animal, mezcla de la unión de los otros ¿Sería una nueva especie?

E: Claro, porque no sería ni salchicha ni de raza.

En relación con la idea de especiación por bibridación hay que señalar que existen modelos de especiación actualmente aceptados basados en la hibridación (FUTUYMA, 2009). Sin embargo, no cabe considerar que las explicaciones encontradas en los estudiantes tengan alguna relación con dichos modelos, que son especialmente complejos.

El razonamiento causal lineal también podría ser un obstáculo subyacente a estas concepciones y explicaciones (GONZÁLEZ GALLI; MEINARDI, 2015; POZO; GÓMEZ CRESPO, 2004), especialmente aquellas basadas en la noción de adaptación (EAAP y EAC), ya que la estructura causal implícita en este tipo de explicaciones puede reconstruirse como sigue, donde cada flecha representa una relación causal: necesidad (representada por algún factor ambiental) $\rightarrow$ cambio individual (mediado por una mutación o el acostumbramiento) $\rightarrow$ cambio evolutivo (adaptación y/o origen de la nueva especie).

En relación con los objetivos de enseñanza, una idea clave es la de divergencia: el hecho de que a partir de un único linaje surjan dos reproductivamente aislados, lo que se representa en las iconografías en forma de "árbol evolutivo" como un "nodo" del que surgen dos ramas. En relación con este objetivo, una cuestión que creemos importante analizar es que solo en un $15 \%$ de las explicaciones dadas como respuesta a la pregunta 3 (aquellas que expresan la idea de EAC) encontramos indicios de que los estudiantes conciban alguna noción de divergencia evolutiva. Por el contrario, el cambio que describen en las demás respuestas se condice más bien con un cambio de tipo anagenético. Si esta interpretación es correcta, dicha explicación no podría dar cuenta del incremento en el número de especies. El siguiente es un ejemplo de lo que interpretamos como expresiones de esta idea de reemplazo de especies:

E: "[...] lo que habia entendido era que cuando una especie no se podia adaptar al medio en que vivia, ponele, creo que los mamuts del frio, después en el calentamiento global se tuvieron que extinguir porque no podian, no podian resistir, ¿Puede ser el calor?’.

I: "Aja".

E: "Y se creó otra, los elefantes, [...] como que se extinguieron ellos [los mamuts] y pasaron a ser los elefantes, que abi si ya estaban adaptados al calor".

Sin embargo, las respuestas basadas en la concepción 9 (divergencia geográfica) revelan algunas ideas que podrían servir de punto de partida para la construcción de un modelo de especiación alopátrico, ya que suponen un evento de divergencia. Sin embargo, estas ideas no se aplican con altos niveles de coherencia. Por ejemplo, una estudiante que en la entrevista manifiesta esta idea expresa también, en otro momento de la misma entrevista, que:

E: "[...] para que dejen de ser ballenas capaz que, por alguna razón, se reproducen con otro animaly se crea otra cosa y dejan de existir las ballenas y empiezan a ser solo esos". 
Este pasaje sugiere la idea reemplazo de una forma por otra. Esta aparente inconsistencia podría interpretarse también como la coexistencia de dos modelos: uno (EAAP) que supone un reemplazo de especies y otro (EAC) que implica la divergencia evolutiva.

En síntesis, de las preguntas 1 y 3 concluimos que las explicaciones más frecuentes que podemos interpretar como basadas en un patrón explicativo para la pregunta sobre el origen de nuevas especies son de dos tipos: (1) basadas en la noción de adaptación a nuevas condiciones ambientales (EAAP y EAC) y (2) por hibridación (EH). Las explicaciones de la especiación basadas en EAAP y EAC son coherentes con el énfasis ya documentado de los estudiantes en las explicaciones adaptativas y teleológicas, mientras que aquellas basadas en la idea de $\mathrm{EH}$ están menos documentadas.

En relación con las concepciones sobre la extinción tenemos dos tipos de respuestas principales: (1) basada en la noción de una adaptación insuficiente y (2) basada en la idea intervención antropogénica. El primer tipo de respuestas es coherente con el énfasis en el factor adaptativo registrado en este trabajo y en muchos otros. La recurrencia a factores antropogénicos a la hora de explicar la extinción no llama la atención dada la gran presencia en los medios de comunicación del tema de la pérdida de biodiversidad como consecuencia de actividades humanas.

En cuanto al concepto de especie utilizado por los estudiantes, interpretamos las categorías estabilidad específica y cambio profundo como la expresión de una concepción según la cual las especies tienen una fuerte estabilidad a través del tiempo de modo que el cambio evolutivo puede implicar un cambio de especie solo cuando la relevancia de los rasgos afectados o la magnitud del cambio supere cierto umbral. Esta concepción podría considerase análoga a un concepto tipológico de especie según el cual cada especie está definida por un conjunto estable de rasgos morfológicos (MAYR, 1998). A partir del análisis de las respuestas a la pregunta 4 inferimos que a mayor semejanza morfológica mayor probabilidad de que los estudiantes asignen los esquemas a una misma especie, lo que también es compatible con un concepto tipológico de especie. Este concepto de especie puede interpretarse como la expresión del obstáculo que denominamos esencialismo de sentido común (ESC) (GELMAN, 2004; GONZÁLEZ GALLI; MEINARDI, 2016), de acuerdo con el cual cada individuo pertenece a una categoría definida por una esencia inmutable. El esencialismo supone que las variaciones interindividuales deben ser más bien leves y superficiales y se las considera como un "ruido de fondo". Podría pensarse que en caso de razonar en base al ESC los estudiantes deberían considerar que no es posible que un conjunto de individuos deje de pertenecer a una especie para pasar a pertenecer a otra. Este podría ser el caso del 53\% que eligió la opción B (cambiarán pero seguirán siendo ballenas). En el caso de los estudiantes que eligieron la opción C (cambiarían y ya no serían ballenas) es posible que consideraran que en ocasiones (cuando el cambio afecta en gran medida al organismo) se puede considerar que la esencia se alteró y que, por lo tanto, ahora se trata de otra especie. Algunas respuestas sugieren que esto sucedería como consecuencia de un factor cuantitativo (el cambio es grande). Por el contrario, otras respuestas sugieren que la diferencia podría ser cualitativa; algunos estudiantes parecen considerar que cuando está involucrada una alteración del material genético (que tendría un estatus causal especial) se justificaría considerar que hay un cambio de especie. Por ejemplo:

E: "Claro, [...] fisicamente los ves parecidos pero tendrías que ver si genéticamente siguen siendo iguales o no". 
Explicaciones, concepciones y obstáculos sobre el origen ...

I: "O sea, para ver si entiendo, ¿Lo interno para vos implicaría algo genético?".

E: "Claro".

I: "Bien, si cambia eso...".

E: "... no sería de la misma especie".

En el mismo sentido, otro estudiante manifiesta que:

I: "¿Qué te parece a vos que tendría que cambiar para que sea otra especie?".

E: "Su genética y el $A D N$ ".

Este estatus especial asignado al material genético es consistente con el gran poder causal que en la cultura popular y en el propio discurso de la biología se le otorga a los genes (KELLER, 2000).

Resumimos estas relaciones entre los niveles jerárquicos de representación en el Cuadro 1.

Cuadro 1. Relación entre patrones explicativos sobre el origen de las especies y el concepto de especie con las principales concepciones y los obstáculos

\begin{tabular}{|c|c|c|c|c|c|c|c|c|c|c|c|c|c|}
\hline \multirow{2}{*}{$\begin{array}{l}\text { Patrones explicativos } \\
\text { y concepto de especie }\end{array}$} & \multicolumn{9}{|c|}{ Concepciones } & \multicolumn{4}{|c|}{ Obstáculos } \\
\hline & 2 & 3 & 4 & 5 & 7 & 8 & 9 & 10 & 11 & TSC & RCL & RCI & ESC \\
\hline EAAP & $\mathrm{X}$ & & $\mathrm{X}$ & $\mathrm{X}$ & & & & & & $\mathrm{X}$ & $\mathrm{X}$ & $\mathrm{X}$ & \\
\hline & & & & & & & & & & & & & \\
\hline EAC & $\mathrm{Y}$ & & $\mathrm{Y}$ & & & & & & & & & & \\
\hline $\mathrm{EAC}$ & $\mathrm{X}$ & & $\mathrm{X}$ & $\mathrm{X}$ & & & $X$ & & & $\mathrm{X}$ & $\mathrm{X}$ & $\mathrm{X}$ & \\
\hline & & & & & & & & & & & & & \\
\hline & & & & & & & & & & & & & \\
\hline & & & & & & & & & & & & & \\
\hline $\mathrm{EH}$ & & $\mathrm{X}$ & $\mathrm{X}$ & $\mathrm{X}$ & & & & & & $\mathrm{X}$ & & $\mathrm{X}$ & \\
\hline & & & & & & & & & & & & & \\
\hline & & & & & & & & & & & & & \\
\hline Concepto de especie & & & & & $\mathrm{X}$ & $\mathrm{X}$ & & & & & & & $\mathrm{X}$ \\
\hline Concepciones sobre extinción & & & & & & & & $\mathrm{X}$ & $\mathrm{X}$ & & & & \\
\hline
\end{tabular}

Fuente: elaborado por los autores.

\section{En relación con investigaciones previas}

Como ya señalamos, son pocas las investigaciones que han explorado este tópico específico. Samarapungavan y Wiers (1997) analizaron las explicaciones de niños de 12 años sobre el origen de las especies y, en coincidencia con nuestros resultados, encuentran un sesgo esencialista en dichas explicaciones. En relación con el patrón explicativo que hemos denominado especiación por cambio adaptativo anagenético profundo (EAAP) estos autores encontraron explicaciones análogas basadas en "cambios microevolutivos adaptativos" (sin embargo, también encuentran explicaciones basadas en las nociones de creación especial y generación espontánea, ausentes en nuestro estudio). 
Por su parte, Evans (2000) analizó explicaciones sobre el origen de las especies en niños de 5 a 12 años encontrando un cambio desde explicaciones basadas en las nociones creacionistas y de generación espontánea para luego dar lugar a un enfoque evolucionista. Este resultado coincide con nuestro hallazgo en relación con la predominancia de la perspectiva evolucionista. Sin embargo, aún en las edades superiores, Evans encuentra una frecuencia relativamente alta de las explicaciones creacionistas. Tal como señalamos, pueden existir importantes diferencias entre poblaciones al respecto según sea la influencia de la cultura religiosa en cada población.

\section{Conclusiones}

En este trabajo buscamos reconstruir los patrones explicativos utilizados por estudiantes de secundaria para explicar el origen de las especies así como el concepto de especie que está implícito en dichas explicaciones. También buscamos identificar qué concepciones específicas aparecen en dichas explicaciones y qué obstáculos epistemológicos podrían subyacer a las mismas. El objetivo de este tipo de estudio interpretativo es aportar a la comprensión de la naturaleza y función de las concepciones de los estudiantes. Es, en definitiva, un intento de ir más allá del "catálogo de errores" de los estudiantes. En relación con las implicancias didácticas sugerimos que este conocimiento constituye una de las condiciones necesarias para un trabajo en las clases que favorezca un mejor aprendizaje de los modelos científicos. Más específicamente, contribuiría a la necesaria reflexión metacognitiva sobre las propias concepciones durante el proceso de construcción los modelos escolares (PETERFALVI, 2001; ZOHAR; DORI, 2012). En cuanto a cómo trabajar en las clases solo recordaremos aquí los lineamientos generales que se prescriben desde el marco teóricos de los obstáculos epistemológicos que suponen (1) favorecer la identificación de los obstáculos, (2) su desestabilización y (3) la reconstrucción conceptual (ASTOLFI; PETERFALVI, 2001). Los análisis que ofrecemos aquí contribuirían sobre todo a los momentos (1) y (2) ya que podrían ayudar a los docentes a diseñar actividades específicamente concebidas para favorecer la explicitación de estas explicaciones y la posterior identificación de las concepciones y obstáculos. Caracterizar y poner un nombre a las concepciones y los obstáculos, así como aprender a reconocerlos en producciones propias y de terceros, es una parte fundamental del aprendizaje. Se trata entonces de un conocimiento sobre algunos aspectos particulares de la cognición de los estudiantes en relación con un contenido específico que constituye un insumo útil para instalar en la aula el "discurso del pensamiento", lo que contribuye a incentivar el desarrollo metacognitivo de los estudiantes. Consideramos que los marcos teóricos de los obstáculos y la metacognición constituyen un encuadre potente para repensar la enseñanza de las ciencias y sugerimos la necesidad de complementarlo con uno basado en los procesos de modelización (en relación con el punto 3 de los lineamientos mencionados) GONZÁLEZ GALLI; MEINARDI, 2016). De todos modos, se requiere profundizar estas investigaciones y, sobre todo, diseñar y llevar a la práctica intervenciones didácticas basadas en estos análisis, como modo último de evaluar su pertinencia y valor para la enseñanza de las ciencias. 


\section{Agradecimientos}

Los autores agradecen al Consejo Nacional de Investigaciones Científicas y Técnicas (CONICET) de Argentina y a la Universidad de Buenos Aires por el financiamiento que hizo posible esta investigación.

\section{Referencias}

ALMEIDA, D. F. Concepções de alunos do ensino médio sobre a origem das espécies. Ciência \& Educação, Bauru, v. 18, n. 1, p. 143-154, 2012. Disponível em: < https://doi. org/10.1590/S1516-73132012000100009>. Acesso em: 20 ago. 2018.

ANDERSON, D.; FISHER, K.; NORMAN, G. Development and evaluation of the conceptual inventory of natural selection. Journal of Research in Science Teaching, Hoboken, v. 39, n. 10, p. 952-978, 2002.

ASTOLFI, J. E1 “error”: un medio para enseñar. Sevilla: Díada, 1999.

ASTOLFI, J.; PETERFALVI, B. Estrategias para trabajar los obstáculos: dispositivos y resortes. In: CAMILLONI, A. (Ed.). Los obstáculos epistemológicos en la enseñanza. Barcelona: Gedisa, 2001. p. 191-223.

BACHELARD, G. La formación del espíritu científico: contribución a un psicoanálisis del conocimiento objetivo. México DF: Siglo XXI, 2004.

COLEY, J. D.; TANNER, K. D. Common origins of diverse misconceptions: cognitive principles and the development of biology thinking. CBE Life Sciences Education, Minneapolis, v. 11, n. 3, p. 209-215, 2012.

COSTA, L. O.; MELO, P. L. C.; TEIXEIRA, F. M. Reflexões acerca das diferentes visões de alunos do ensino médio sobre a origem da diversidade biológica. Ciência \& Educação, Bauru, v. 17, n. 1, p. 115-128, 2011. Disponível em: <https://doi.org/10.1590/S151673132011000100008>. Acesso em: 20 ago. 2018.

EVANS, M. The emergence of beliefs about the origins of species in school-age children. Merrill Palmer Quarterly, Detroit, v. 46, n. 2, p. 221-254, 2000.

FUTUYMA, D. Evolution. Sunderland: Sinauer, 2009.

GELMAN, S. Psychological essentialism in children. Trends in Cognitive Sciences, Cambridge, v. 8, n. 9, p. 404-409, 2004.

GLASER, B.; STRAUSS, A. The discovery of grounded theory: strategies for qualitative research. New York: Aldine, 1967.

GONZÁLEZ GALLI, L.; MEINARDI, E. ¿Está en crisis el darwinismo?: los nuevos modelos de la biología evolutiva y sus implicaciones didácticas. Didáctica de las Ciencias Experimentales y Sociales, Valencia, n. 27, p. 219-234, 2013. 
Galli, L. M. G.; Pérez, G. M.; Alegre, C. K.; Escoto, S. J.

GONZÁLEZ GALLI, L.; MEINARDI, E. Obstáculos para el aprendizaje del modelo de evolución por selección natural. In: CUVI, N. et al. (Ed.). Evolucionismo en América y Europa: antropología, biología, política y educación. Quito: Doce Calles: Flacso, 2016. p. 463-476.

GONZÁLEZ GALLI, L.; MEINARDI, E. Obstáculos para el aprendizaje del modelo de evolución por selección natural en estudiantes de escuela secundaria de Argentina.

Ciência \& Educação, Bauru, v. 21, n. 1, p. 101-122, 2015. Disponível em: <https://doi. org/10.1590/1516-731320150010007>. Acesso em: 22 ago. 2018.

GONZÁLEZ GALLI, L.; MEINARDI, E. The role of teleological thinking in learning the Darwinian model of evolution. Evolution: Education and Outreach, New York, v. 4, n. 1, p. 145-152, 2011.

GOULD, S. La estructura de la teoría evolutiva. Barcelona: Tusquets, 2004.

INAGAKI, K.; HATANO, G. Young children's conception of the biological world. Current Directions in Psychological Science, Thousand Oaks, v. 15, n. 4, p. 177-181, 2006.

JIMÉNEZ ALEIXANDRE, M. Cambiando las ideas sobre el cambio biológico. Enseñanza de las Ciencias, Barcelona, v. 9, n. 3, p. 248-256, 1991.

KAMPOURAKIS, K. Understanding evolution. Cambridge: Cambridge University Press, 2014.

KELLER, E. F. Lenguaje y vida: metáforas de la biología en el siglo XX. Buenos Aires: Manatial, 2000.

LATORRE BELTRÁN, A.; RINCÓ IGEA, D.; ARNAL, A. Bases metodológicas de la investigación educativa. Barcelona: Hurtado, 1997.

LLOYD, E. The structure and confirmation of evolutionary theory. Princeton: Princeton University Press, 1994.

MAYR, E. Así es la biología. Madrid: Debate, 1998.

PETERFALVI, B. Identificación de los obstáculos por parte de los alumnos. In: CAMILLONI, A. (Ed.). Los obstáculos epistemológicos en la enseñanza. Barcelona: Gedisa, 2001. p. 127-168.

POZO, J.; GÓMEZ CRESPO, M. Aprender y enseñar ciencia. Madrid: Morata, 2004.

SAMARAPUNGAVAN, A.; WIERS, R. Children's thougths on the origin of species: a study on explanatory coherence. Cognitive Science, New York, v. 21, n. 2, p. 147-177, 1997.

SELLES, S. E.; DORVILLE, L. F. M.; PONTUAL, L. V. Ensino religioso nas escolas estaduais de Rio de Janeiro: implicações para o ensino de ciências/biología. Ciência \& Educação, Bauru, v. 22, n. 4, p. 875-894, 2016. Disponível em: <http://dx.doi. org/10.1590/1516-731320160040004>. Acesso em: 20 ago. 2018.

SMITH, M. Current status of research in teaching and learning evolution: II. pedagogical issues. Science and Education, London, v. 19, n. 4-8, p. 523-538, 2010. 
STAMOS, D. Evolution and the big questions: sex, race and other matters. Malden: Blackwell, 2008.

STERELNY, K.; GRIFFITHS, P. Sex and death: an introduction to philosophy of biology. Chicago: The University of Chicago Press, 1999.

TALANQUER, V. On cognitive constraints and learning progressions: the case of "structure of matter". International Journal of Science Education, Abingdon, v. 31, n. 15, p. 2123-2136, 2009.

THOMPSON, P. The structure of biological theories. Albany: State University of New York Press, 1989.

ZOHAR, A.; DORI, Y. (Ed.). Metacognition in science education: trends in current research. Dordretch: Springer. 2012.

Anexo A. Instrumento utilizado para la toma de datos

(1) El león, la mosca, la ballena, la bacteria, el hongo y la planta de maíz (el choclo) son 6 especies de seres vivos. Los científicos creen que, en total, hay entre 10 y 100 millones de especies ¿Cuál creés que es el origen de cada una de estas especies? Marcá la opción con la que estás más de acuerdo haciendo una cruz en la columna de la derecha.

\begin{tabular}{|l|l|l|}
\hline A & Cada especie surgió de un modo natural e independiente. & \\
\hline B & Cada una de estas especies fue creada por algo o alguien. & \\
\hline C & Cada especie surgió por modificación de otra especie anterior. & \\
\hline
\end{tabular}

Explicá por qué elegiste esa opción.

(2) Si estudiáramos a los descendientes de las ballenas dentro de 10 millones de años ¿Creés que serían iguales? ¿Seguirían siendo ballenas? Las siguientes son respuestas de alumnos de tu edad de otros colegios. Marcá con una cruz la opción con la que estés más de acuerdo.

\begin{tabular}{|l|l|l|}
\hline A & Creo que no cambiarían, serían iguales. Seguirían siendo ballenas. & \\
\hline B & Creo que cambiarían pero seguirían siendo ballenas. & \\
\hline C & Creo que cambiarían y ya no serían ballenas, serían otra especie. & \\
\hline
\end{tabular}

Explicá por qué elegiste esa opción. 
(3) Los científicos creen que hace unos 10 millones de años solo había una única especie de felino. Luego, hace unos 5 millones de años, había unas 100 especies. Actualmente solo hay unas 40 especies, entre ellos el tigre, el león y la pantera ¿Cómo creés que llegaron a existir 100 especies de felinos si antes había solo había una? ¿Por qué creés que actualmente solo hay unas 40 especies si antes existieron unas 100 ?

(4) El siguiente esquema muestra distintos momentos de la evolución de un animal. El dibujo muestra cómo cambió el aspecto y tamaño de estos animales a través miles de años según la reconstrucción de los paleontólogos a partir de los fósiles.
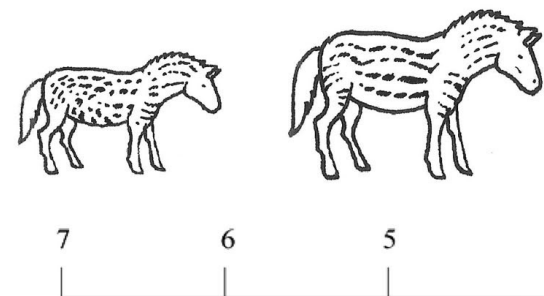

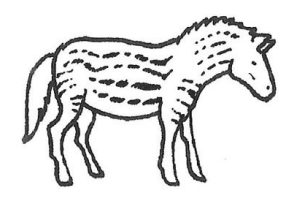

3

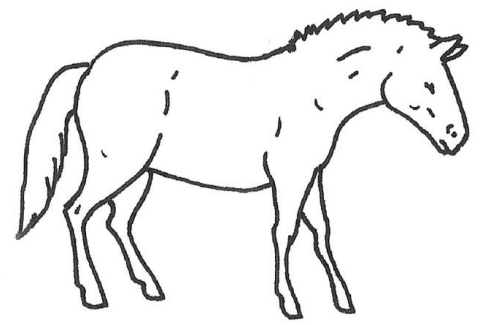

Tiempo (millones de años antes del presente)

Completá el siguiente cuadro poniendo SI o NO en la columna del medio y explicá el porqué de tu respuesta en la columna de la derecha:

\begin{tabular}{|l|l|l|}
\hline ¿Te parece que (A) y (B) pertenecen a la misma especie? & & \\
\hline ¿Te parece que (B) y (C) pertenecen a la misma especie? & & \\
\hline$¿$ Te parece que (C) y (D) pertenecen a la misma especie? & & \\
\hline$¿$ ¿Te parece que (A) y (D) pertenecen a la misma especie? & & \\
\hline
\end{tabular}

Artigo recebido em 26/04/2017. Aceito em 16/01/2018.

Contacto: Consejo Nacional de Investigaciones Cientificas y

Tecnicas, Godoy Cruz, 2290, Buenos Aires C1425FQB, Argentina. 